\title{
Acquired Long QT Syndrome and Torsades de Pointes after Mitral Valve Replacement Surgery
}

Carlos Rodríguez Artuza ${ }^{1,2, *}$, Héctor Barajas², José Rivera², Emilio Clavel², Mayela Labarca²

ORCID IDs

Artuza CR (D) https://orcid.org/0000-0001-7925-6145
Barajas H (D) https://orcid.org/0000-0002-2704-3807

Labarca M (D) https://orcid.org/0000-0002-2789-3537

\begin{abstract}
Acquired long QT syndrome (aLQTS) can occur in up to one third of patients undergoing cardiac surgery and is often undisclosed. We present a case of a 55-year-old male patient admitted to our center for mitral valve replacement surgery, and, during the postoperative period, a long QT greater than 600 ms was confirmed and in the Holter monitoring Torsade de Pointes (TdP) was evidenced. The patient received appropriate medical treatment and was discharge in stable clinical conditions.
\end{abstract}

KEYWORDS: Torsades de Pointes; Ventricular tachyarrhythmias; Postoperative period.

\section{INTRODUCTION}

Acquired long QT syndrome (aLQTS) can occur in up to a third of patients undergoing cardiac surgery and is often unrecognized. A prolonged QT greater than $500 \mathrm{~ms}$ implies high risk of triggering Torsade de Pointes (TdP), ventricular fibrillation (VF) and sudden death. Among the pathophysiological mechanisms involved, we have:

- Generalized inflammatory reaction;

- Adrenaline release;

- Use of antibiotics;

- Use of anesthetics;

- Use of antiarrhythmic drugs;

- Advanced age;

- Female gender;

- Polypharmacy;

- Electrolyte imbalance;

- Previous heart disease ${ }^{1-5}$.

1. Universidad del Zulia - Facultad de Medicina - Instituto de Investigaciones de Enfermedades Cardiovasculares - Maracaibo/Zulia - Venezuela.

2. Centro Clínico La Sagrada Familia - Medicine - Cardiology - Maracaibo/Zulia - Venezuela.

*Corresponding author: cardiocarral@gmail.com

Received: Feb 22, 2021 | Accepted: Apr 24, 2021 


\section{CLINICAL CASE}

A 55-year-old male patient with diagnosis of severe mitral regurgitation and paroxysmal atrial fibrillation (PAF) underwent mitral valve replacement due to evolving with dyspnea and progressive deterioration of his functional class with preserved ventricular function. The patient denies a history of syncope and, prior to admission, developed an episode of palpitations and PAF that resolved on an outpatient basis.

On the fifth day after cardiac surgery, a Holter monitor was placed for sudden periods of palpitations and blurred vision. During monitoring, aLQTS of 610 ms was documented (Fig. 1), and VF deflagrated by an R on T phenomenon (Fig. 2) induced TdP and VF was triggered. Patient was successfully defibrillated, and amiodarone and ciprofloxacin were discontinued due to chronic use for paroxysmal atrial fibrillation as an antimicrobial post-surgical protocol. We started ventricular pacing at a rate of 85 beats per minute with a temporary VVI pacemaker (Fig. 3) for two days and administered magnesium sulfate even with serum electrolytes within the normal range.

Patient was discharged ten days after surgery and during a one-year follow-up evolved clinically stable with NYHA I functional class, sinus rhythm, normal QTc interval and caring not to use drugs that could potentially lengthen the QTc interval.

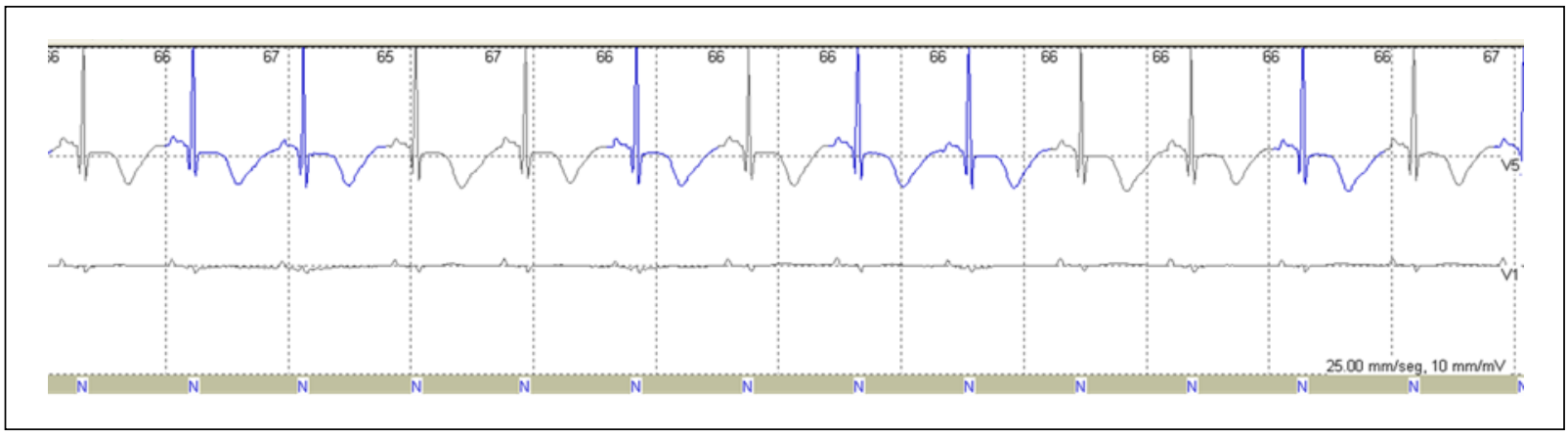

Figure 1. Acquired long QT syndrome with a QTc of $610 \mathrm{~ms}$.

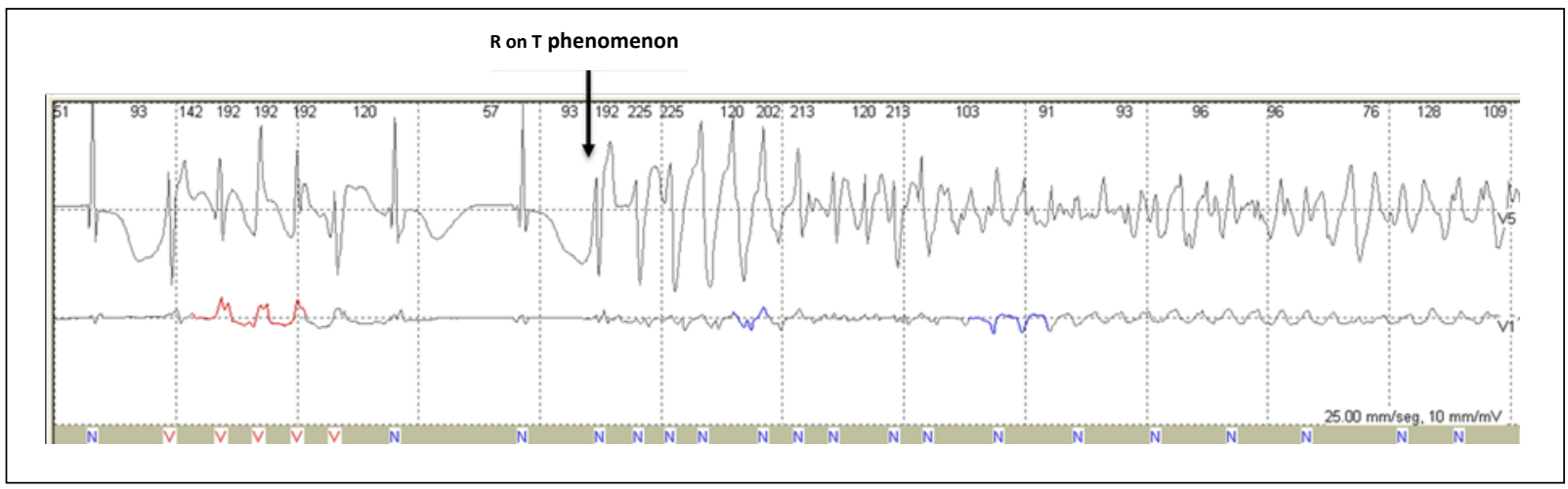

Figure 2. Ventricular fibrillation deflagrated by an R on T phenomenon that induces Torsade de Pointes.

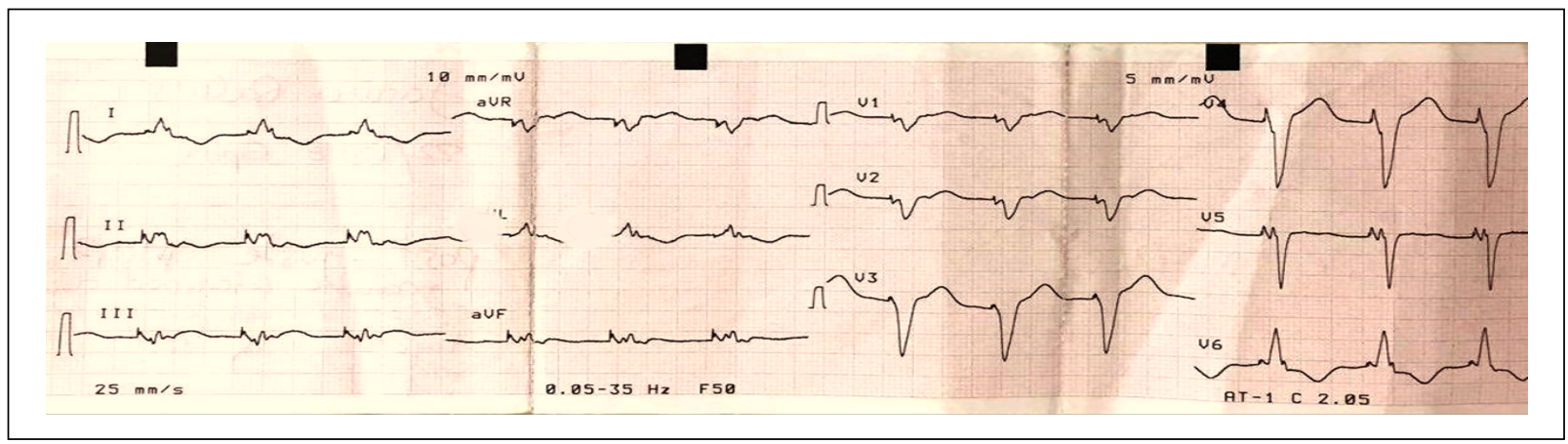

Figure 3. Ventricular pacing at a rate of 85 beats per minute. 


\section{DISCUSSION}

Up to a third of patients undergoing cardiac surgery may present aLQTS during the postoperative period. However, $\mathrm{TdP}$ is an infrequent phenomenon. Sometimes when the QTc is prolonged over $500 \mathrm{~ms}$, it can trigger TdP and VF. The current patient lengthened the QTc to $610 \mathrm{~ms}^{1}$. In addition, several risk factors associated with the appearance of TdP are known, but nowadays the exact mechanism by which some patients deplete the ventricular repolarization reserve and others don't is unknown.

In this case, we present a 55-year-old male patient who underwent a surgery for treating severe mitral regurgitation. Recently, a risk score for QTc prolongation associated with drugs has been validated, in which age over 50 years is related as an indicator of risk for developing aLQTS ${ }^{6}$. Cardiac surgery is a large-scale procedure in which there is an acute posttraumatic inflammatory response that triggers the release of cytokines that act directly on cardiomyocytes architecture, causing an acquired channelopathies by prolonging the action potential and inducing LQTS.

On the other hand, the inflammatory mechanism itself exerts a direct action on the central nervous system that generates the release of adrenaline, favoring the appearance of $\mathrm{TdP}^{2}$. Antibiotics, anesthetic and antiarrhythmic agents have been implicated as triggers of TdP in susceptible patients. In this patient, servoflurane was used; we had to discontinue amiodarone and ciprofloxacin. It is believed that drugs could trigger TdP by unmasking subclinical genetic channelopathies or by directly altering ion channels and depleting the ventricular repolarization reserve ${ }^{3}$.

The sum of the inflammatory mechanism, the adrenergic discharge, the age of the patient, the use of servoflurane, amiodarone, ciprofloxacin, and mitral regurgitation contributed to the appearance of aLQTS, TdP and VF in this patient, who was successfully defibrillated. Even though his serum electrolytes were normal, he received intravenous magnesium sulfate ${ }^{7}$. Ventricular pacing at a heart rate of 85 beats per minute is effective for treating TdP due to aLQTS, homogenizing the $\mathrm{R}-\mathrm{R}$ interval and reducing ventricular refractoriness dispersion. On this basis, we opted for the implantation of a temporary pacemaker and then wean the artificial ventricular pacing 48 hours later ${ }^{7,8}$.

It is believed that aLQTS is produced by multiple causes and that there is a certain genetic susceptibility for developing it ${ }^{9}$. In this patient, after eliminating all the drugs that lengthen the QTc and once the inflammatory phase has disappeared, he has never presented abnormal QTc again. The patient was discharged asymptomatic and in stable clinical conditions, emphasizing not to use drugs that prolong the QTc interval. Holter and control electrocardiogram are performed periodically with QTc interval measurements.

\section{CONCLUSION}

We must be careful during the postoperative period of all patients undergoing cardiac surgery since, due to the previous heart disease, these patients are at risk of triggering TdP. The administration of drugs must be monitored taking into account which drugs can potentially prolong the QT interval. Finally, all of these patients should be closely monitored for warning symptoms of hemodynamic decompensation.

\section{REFERENCES}

1. Biry M, Schurr U, Ritter S, Baenziger K, Zollinger A, Genoni M. High incidence of severely prolonged QT interval after cardiac surgery. Crit Care. 2010;14(Suppl. 1):P134. https://doi.org/10.1186/cc8366

2. $\quad$ Tong KL, Lau YS, Teo WS. A case series of drug-induced long QT syndrome and torsade de pointes. Singapore Med J. 2001;42(12):566-70.

3. Lazzerini PE, Capecchi PL, Laghi-Pasini F. Long QT syndrome: an emerging role for inflammation and immunity. Front Cardiovasc Med. 2015;2:26. https://doi.org/10.3389/fcrm.2015.00026 
4. Gilbert S, Singh D, Jesuraj ML. Long QT syndrome and torsades de pointes complicating mitral valve replacement. Indian Heart J. 2016;68(Suppl. 2):S210-S211. https://doi.org/10.1016/j.ihj.2015.07.015

5. Lazzerini PE, Capecchi PL, El-Sherif N, Laghi-Pasini F, Boutjdir M. Emerging Arrhythmic Risk of Autoimmune and Inflammatory Cardiac Channelopathies. J Am Heart Assoc. 2018;7(22):e010595. https://doi.org/10.1161/JAHA.118.010595

6. Berger FA, van der Sijs H, Becker ML, van Gelder T, van den Bemt PMLA. Development and validation of a tool to assess the risk of QT drug-drug interactions in clinical practice. BMC Med Inform Decis Mak. 2020;20(1):171. https://doi.org/10.1186/s12911-02001181-3

7. Pinski SL, Eguía LE, Trohman RG. What is the minimal pacing rate that prevents torsades de pointes? Insights from patients with permanent pacemakers. Pacing Clin Electrophysiol. 2002;25(11):1612-5. https://doi.org/10.1046/j.1460-9592.2002.01612.x

8. Drew BJ, Ackerman MJ, Funk M, Gibler WB, Kligfield P, Menon V, et al. Prevention of torsade de pointes in hospital settings: a scientific statement from the American Heart Association and the American College of Cardiology Foundation. J Am Coll Cardiol. 2010;55(9):934-47. https://doi.org/10.1016/j.jacc.2010.01.001

9. Mahida S, Hogarth AJ, Cowan C, Tayebjee MH, Graham LN, Pepper CB. Genetics of congenital and drug-induced long QT syndromes: current evidence and future research perspectives. I Interv Card Electrophysiol. 2013;37:9-19. https://doi.org/10.1007/s10840-0139779-5 